\title{
Evaluación del uso de trampa de evaporación en la calibración de pipetas de pistón en volúmenes menores o iguales a $100 \boldsymbol{\mu l}$
}

\section{Evaluation of evaporation trap on the calibration of piston pipettes within nominal values under or equal to $100 \mu \mathrm{l}$}

\author{
Johanna Méndez Arias \\ Escuela Ingeniería Industrial e Instituto de Investigaciones en Ingeniería \\ Universidad de Costa Rica \\ José Eduardo Marín \\ Laboratorio de Metrología, Normalización y Calidad, \\ Instituto de Investigaciones en Ingeniería \\ Universidad de Costa Rica
}

Recibido: 14 de marzo 2017

Aceptado: 3 de agosto 2017

\begin{abstract}
Resumen
El presente documento tiene como objetivo demostrar la importancia de utilizar trampa de evaporación durante el proceso de calibración de pipetas de pistón, específicamente a niveles de microlitros $(\mu \mathrm{L})$. Se realizaron calibraciones a una pipeta del Laboratorio de Metrología, Normalización y Calidad (LABCAL) mediante el método gravímetro, con trampa de evaporación y sin utilizar trampa ni corrección por evaporación. Se realizaron 10 repeticiones en tres puntos diferentes y los valores fueron comparados con un valor de referencia proporcionado por el certificado de calibración de la misma pipeta analizada, el cual es determinado por el Laboratorio Nacional de Metrología (LACOMET). Como resultado se demuestra que al no utilizar trampa de evaporación se obtienen diferencias de hasta $1 \mu \mathrm{L}$ con respecto al valor de referencia.
\end{abstract}

\section{Palabras clave}

Volumen, calibración, trampa de vapor, metrología, evaporación.

\begin{abstract}
This paper aims to demonstrate the importance of using evaporation trap during piston pipette calibration, specifically at microliter levels $(\mu \mathrm{L})$. Calibrations were performed in a laboratory pipette using the gravimeter method with evaporation trap, and without trap or any correction by evaporation. 10 repetitions were performed at three different points and the values provided by the calibration certificate of the analyzed pipette were compared to a reference value determined by the National Metrology Laboratory (LACOMET).
\end{abstract}

Keywords

Volume, calibration, steam trap, metrology, evaporation. 


\section{INTRODUCCIÓN}

Para análisis químicos, microbiológicos, médicos y farmacéuticos, entre otros, es de vital importancia conocer con gran exactitud los volúmenes dispensados por las pipetas de pistón, donde desviaciones de valores a nivel de microlitros pueden generar resultados falsos positivos o negativos, involucrando diagnósticos equivocados que podrían incluso afectar vidas $(1,2)$.

La calibración de instrumentos relacionados a la magnitud volumen puede ser realizada por diferentes métodos: geométrico, volumétrico y gravimétrico. El método gravimétrico es comúnmente el más utilizado en la calibración de pipetas de pistón; este método es indirecto y consiste en determinar el volumen dispensado por la pipeta utilizando una sustancia de densidad conocida mediante un instrumento de pesar $(3,4)$.

Según la ISO 8656-2002, para la calibración de pipetas de pistón de volúmenes menores a 50 es recomendable utilizar trampa de evaporación para disminuir los efectos de evaporación presentes en un proceso de calibración que podrían afectar el resultado final y la incertidumbre asociada.

La trampa de evaporación consiste en un recipiente cóncavo colocado alrededor del plato de la balanza que se llena con agua con el fin de saturar el ambiente y de esta forma evitar que parte del agua dispensada en el recipiente receptor se escape al ambiente, afectando la estabilidad y el resultado de medición.

El objetivo de este artículo es comparar los resultados de la calibración de una pipeta de pistón en diferentes puntos $(10 \mu \mathrm{L}, 50 \mu \mathrm{L}$ y $100 \mu \mathrm{L})$, mediante el método gravimétrico, con y sin la utilización de trampa de evaporación acoplada a los instrumentos de pesar, contrastando los resultados en relación al error y la incertidumbre asociada al proceso de medición.

\section{METODOLOGÍA}

Se compararon los resultados obtenidos en un proceso de calibración de una pipeta de pistón del LABCAL, realizando el proceso con y sin trampa de evaporación, tomando como referencia valores proporcionados por LACOMET; se calibraron tres puntos diferentes $(10 \mu \mathrm{L}, 50 \mu \mathrm{L}$ y $100 \mu \mathrm{L})$, realizando 10 repeticiones en cada punto, determinando el error de medición y la incertidumbre asociada; se realizaron correcciones por empuje del aire, correcciones en la densidad del agua por gases disueltos y fugacidad, corrección por efecto de la temperatura y se consideró la resolución de la pipeta de pistón y la repetibilidad del proceso en la estimación de la incertidumbre.

\subsection{Método de calibración}

Las pruebas se realizaron utilizando el método gravimétrico con el procedimiento LABCAL-PC-V-01, aplicado con y sin trampa de evaporación. Las características metrológicas y la trazabilidad de los equipos utilizados se presentan en las tablas 1 y 2. 
Tabla 1. Características metrológicas del equipo utilizando en el proceso de calibración.

\begin{tabular}{ccc}
\hline Equipo & Resolución & $\begin{array}{c}\text { Incertidumbre } \\
\text { expandida }(\mathrm{k}=2)\end{array}$ \\
\hline Balanza & $0,000001 \mathrm{~g}$ & $0,0000017 \mathrm{~g}$ \\
$\begin{array}{c}\text { Medidor de condiciones } \\
\text { ambientales }\end{array}$ & $0,5^{\circ} \mathrm{C}$ & $0,6{ }^{\circ} \mathrm{C}$ \\
& $1 \mathrm{hPa}$ & $2 \mathrm{hPa}$ \\
Termopar & $1 \%$ & $2 \%$ \\
\hline
\end{tabular}

Tabla 2. Datos de trazabilidad de los instrumentos utilizados en las calibraciones.

\begin{tabular}{ccc}
\hline Instrumento & Certificado & Trazabilidad \\
\hline Balanza & 11030312 & LACOMET \\
Termopar & $20120927-46-13$ & SCM Metrología \\
$\begin{array}{c}\text { Medidor de condiciones } \\
\text { ambientales }\end{array}$ & 1129071211701012 & LACOMET \\
\hline
\end{tabular}

\subsection{Modelo Matemático}

$$
V_{20}=\left(M_{L}-M_{v c}\right)\left(1-\frac{\rho_{A}}{\rho_{p}}\right)\left(\frac{1}{\rho_{w}-\rho_{A}}\right)\left[1-\gamma\left(t_{w}-t_{r}\right)\right]
$$

donde:

$\mathrm{V}_{20}=$ Volumen vertido referido a $20^{\circ} \mathrm{C}\left(\mathrm{cm}^{3}\right)$;

$\mathrm{M}_{\mathrm{L}}=$ Masa recipiente lleno $(\mathrm{g})$;

$\mathrm{M}_{\mathrm{vc}}=$ Masa recipiente vacio $(\mathrm{g})$;

$\varrho_{\mathrm{w}}=$ Densidad del agua $\left(\mathrm{g} / \mathrm{cm}^{3}\right)$;

$\varrho_{A}=$ Densidad del aire $\left(\mathrm{g} / \mathrm{cm}^{3}\right)$;

$\varrho_{\mathrm{p}}=$ Densidad de la pesa de referencia $\left(\mathrm{g} / \mathrm{cm}^{3}\right)$;

$\gamma=$ Coeficiente de dilatación cúbica del material de la punta utilizada durante el proceso de calibración $\left({ }^{\circ} \mathrm{C}-1\right)$;

$\mathrm{t}_{\mathrm{w}}=$ Temperatura del agua $\left({ }^{\circ} \mathrm{C}\right)$;

$\mathrm{t}_{\mathrm{r}}=$ Temperatura de referencia $\left({ }^{\circ} \mathrm{C}\right)$, en este caso se utilizará $20^{\circ} \mathrm{C}$; 


\section{RESULTADOS}

El error determinado en cada punto y las diferencias obtenidas con respecto al valor de referencia se muestran en la tabla 3.

Tabla 3. Errores obtenidos en los procesos de calibración y sus diferencias respecto al valor de referencia utilizado.

\begin{tabular}{ccccc}
\hline & \multicolumn{2}{c}{ Resultado } & \multicolumn{2}{c}{ Diferencias } \\
\cline { 2 - 5 } $\begin{array}{c}\text { Referencia, REF } \\
(\mu \mathrm{L})\end{array}$ & $\begin{array}{c}\text { Con } \\
\text { trampa, CT } \\
(\mu \mathrm{L})\end{array}$ & $\begin{array}{c}\text { Trampa, ST } \\
(\mu \mathrm{L})\end{array}$ & REF -CT & REF -ST \\
10,14 & 10,18 & 9,95 & $-0,04$ & 0,19 \\
49,53 & 49,57 & 48,90 & $-0,04$ & 0,63 \\
99,50 & 99,53 & 98,49 & $-0,03$ & 1,01 \\
\hline
\end{tabular}

Como se puede observar en la tabla 3 los resultados obtenidos sin utilizar trampa de evaporación presentan mayores desviaciones con respecto al valor de referencia, con desvíos de hasta $1 \mu \mathrm{L}$, que en ese caso reportan valores dispensados por la pipeta menores a los que realmente dispensó. Ocurre esto porque no hubo ninguna compensación por las pérdidas de evaporación, ni mecanismo para minimizarla. Dicha evaporación se ve reflejada en un valor menor de volumen determinado como dispensado por la pipeta en comparación con el valor obtenido cuando se utilizó trampa de evaporación. Es importante resaltar que las condiciones ambientales fueron estables durante los procesos de calibración como se muestra en la tabla 4.

Tabla 4. Condiciones ambientales durante el proceso de calibración de la pipeta de pistón.

\begin{tabular}{|c|c|c|c|c|c|c|c|c|}
\hline & \multicolumn{2}{|c|}{ Temperatura $\left({ }^{\circ} \mathrm{C}\right)$} & \multicolumn{2}{|c|}{$\begin{array}{l}\text { Humedad Relativa } \\
(\%)\end{array}$} & \multicolumn{2}{|c|}{$\begin{array}{l}\text { Presión Atmosférica } \\
\qquad(\mathrm{hPa})\end{array}$} & \multicolumn{2}{|c|}{$\begin{array}{l}\text { Temperatura } \\
\text { del agua }\left({ }^{\circ} \mathrm{C}\right)\end{array}$} \\
\hline & Inicial & Final & Inicial & Final & Inicial & Final & Inicial & Final \\
\hline $10 \mu \mathrm{L}$ con trampa & 20,5 & 21,0 & 53 & 53 & 875 & 875 & 19,5 & 19,6 \\
\hline $10 \mu \mathrm{L} \sin$ trampa & 19,5 & 20,0 & 54 & 51 & 879 & 876 & 18,0 & 18,6 \\
\hline $50 \mu \mathrm{L}$ con trampa & 20,0 & 20,0 & 52 & 55 & 878 & 875 & 19,6 & 19,6 \\
\hline $50 \mu \mathrm{L} \sin$ trampa & 21,0 & 20,5 & 58 & 55 & 876 & 875 & 19,7 & 19,2 \\
\hline $100 \mu \mathrm{L}$ con trampa & 20,0 & 20,5 & 57 & 55 & 875 & 875 & 19,2 & 19,3 \\
\hline $100 \mu \mathrm{L}$ sin trampa & 20,5 & 20,0 & 55 & 54 & 875 & 879 & 19,4 & 18,9 \\
\hline
\end{tabular}

Para tener una visión más clara de las diferencias se puede observar en la Fig. 1 un gráfico de desviación de los valores en ambas situaciones. 


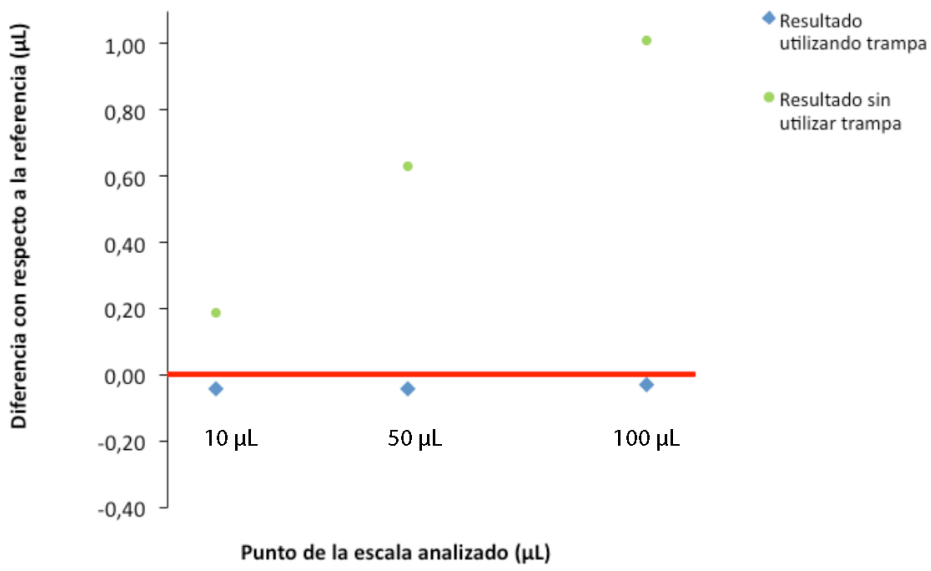

Figura 1. Desviaciones con respecto a un valor de referencia del resultado de calibración de una pipeta de pistón utilizando y sin utilizar trampa de evaporación.

Es importante considerar que el valor de referencia tiene una incertidumbre asociada, por lo que la tolerancia en la comparación de los errores estaría en un intervalo: valor de referencia \pm incertidumbre expandida, utilizando $95,45 \%$ de confianza para un factor de cobertura $\mathrm{k}=2$.

Considerando como límite superior el valor de referencia más la incertidumbre expandida asociada y como límite inferior el valor de referencia menos la incertidumbre expandida asociada, se graficaron los valores de volumen determinados en el proceso de calibración en ambos casos, con y sin trampa de evaporación, para determinar si estaban entre los intervalos asociados a la referencia; los resultados se muestran en las figuras 2,3 y 4 .

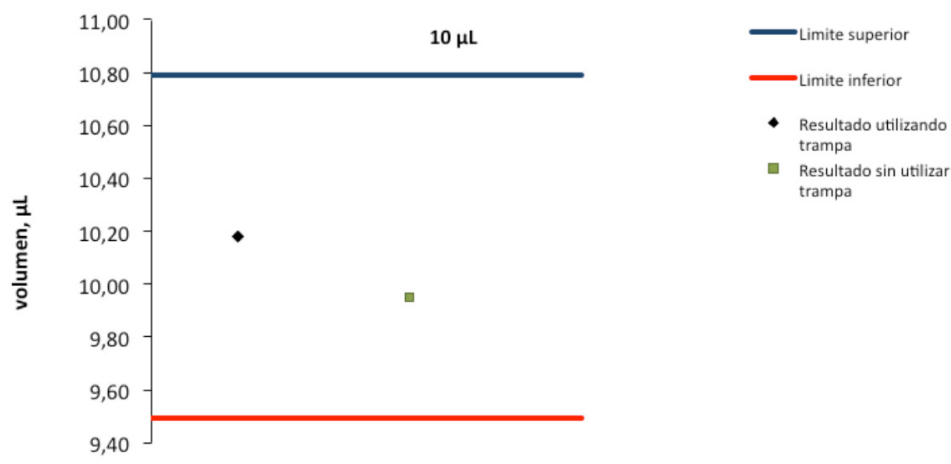

Figura 2. Resultados de error obtenidos de la calibración de la pipeta de pistón con y sin utilizar trampa de evaporación, en el punto de $10 \mu \mathrm{L}$. 
92 MÉNDEZ Y MARÍN: Evaluación del uso de trampa de evaporación en la calibración de pipetas de pipetas...

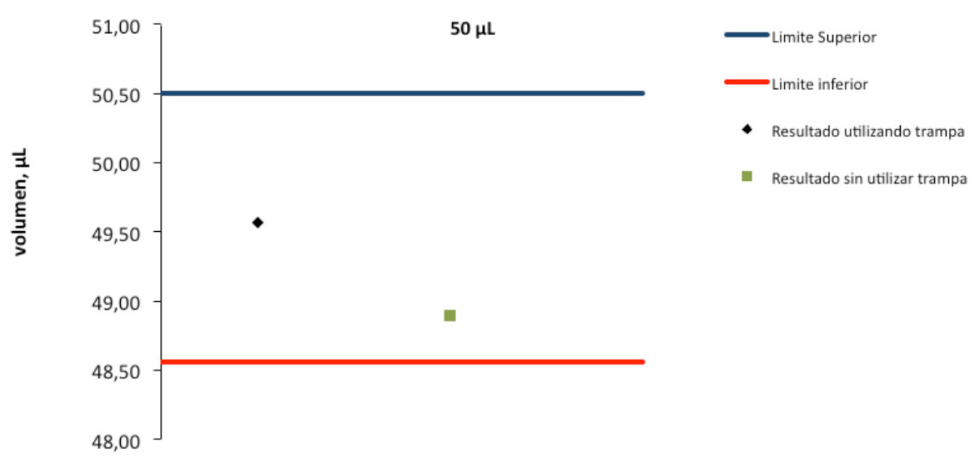

Figura 3. Resultados de error obtenidos de la calibración de la pipeta de pistón con y sin el uso de trampa de evaporación, en el punto de $50 \mu \mathrm{L}$.

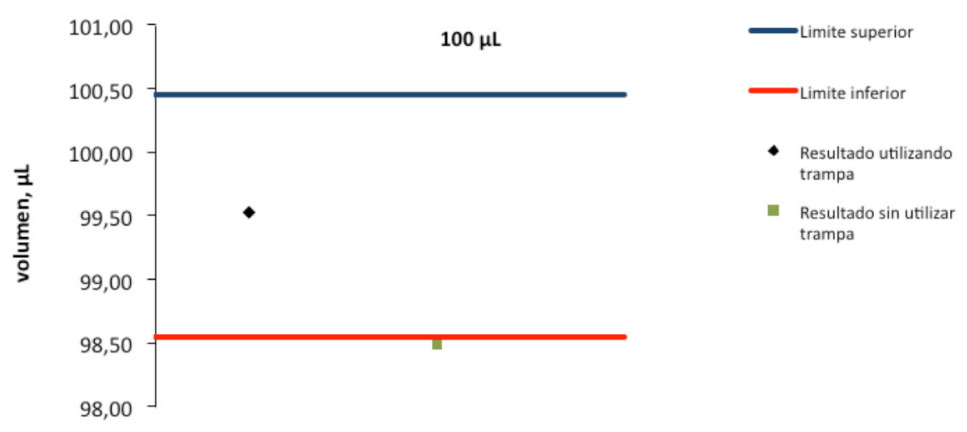

Figura 3. Resultados de error obtenidos de la calibración de la pipeta de pistón con y sin el uso de trampa de evaporación, en el punto de $100 \mu \mathrm{L}$.

Se puede observar que los valores obtenidos sin utilizar trampa de evaporación están más cercanos al límite inferior, reflejando así las pérdidas debidas a evaporación y no contempladas, siendo más evidente en volúmenes de $100 \mu \mathrm{L}$, donde el valor medio estaría totalmente fuera de los limites de referencia.

Sin embargo, es vital dentro de la metrología y en mediciones de alta exactitud considerar la incertidumbre asociada al proceso de medición. Por lo tanto, considerando dicha incertidumbre a un porcentaje de confianza del 95,45\% de confianza y a un factor de cobertura $\mathrm{k}=2$, se obtuvieron los resultados mostrados en las figuras 4,5 y 6 . 


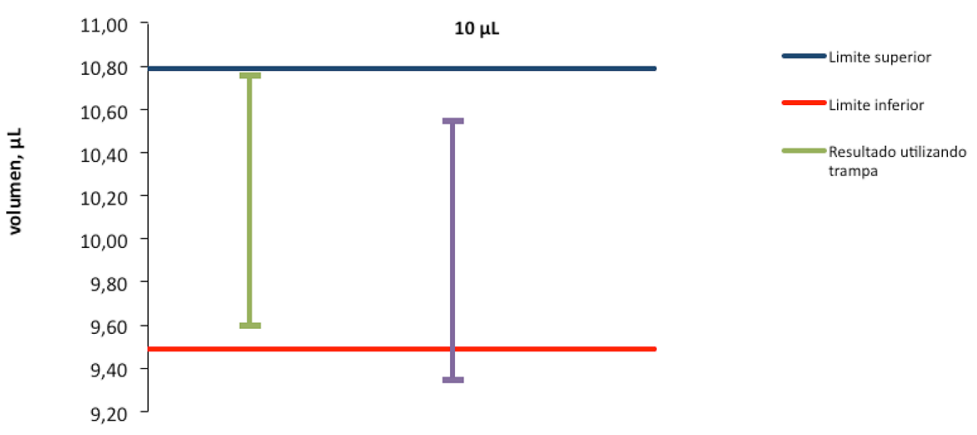

Figura 4. Resultados de error considerando la incertidumbre de la medición obtenidos de la calibración de la pipeta de pistón con y sin utilizar trampa de evaporación, en el punto de $10 \mu \mathrm{L}$.

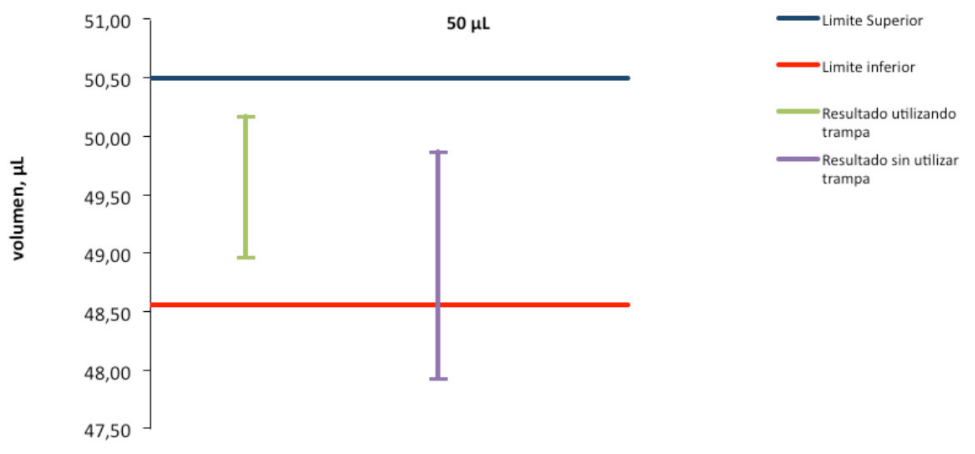

Figura 5. Resultados de error considerando la incertidumbre de la medición obtenidos de la calibración de la pipeta de pistón con y sin trampa de evaporación, en el punto de $50 \mu \mathrm{L}$.

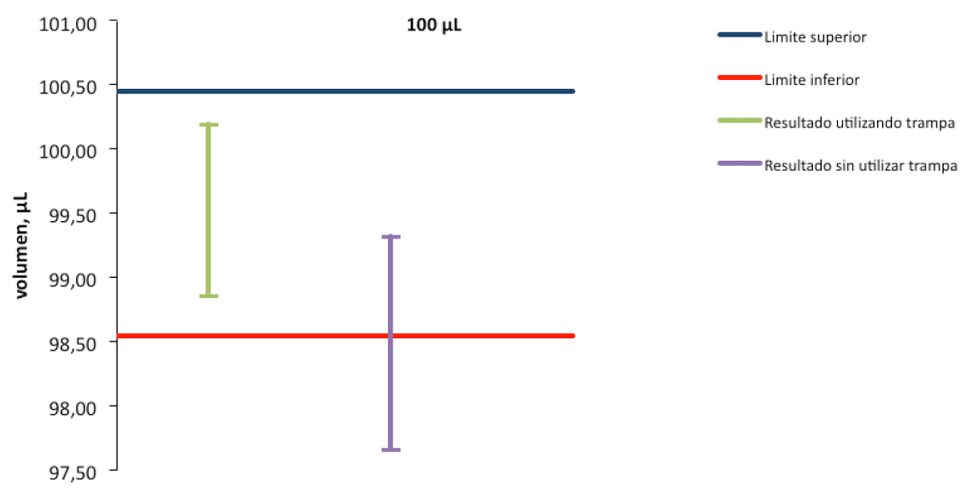

Figura 6. Resultados de error considerando la incertidumbre de la medición obtenidos de la calibración de la pipeta de pistón con y sin trampa de evaporación, en el punto de $100 \mu \mathrm{L}$. 
Se puede observar en las figuras 4,5,6, que incluyen los valores de la estimación de la incertidumbre en la determinación del volumen dispensado por una pipeta de pistón de $10 \mu \mathrm{L}$ a $100 \mu \mathrm{L}$ a una temperatura normalizada de referencia de $20^{\circ} \mathrm{C}$, que los resultados obtenidos con el uso de la trampa de evaporación se encuentran en conformidad con los límites de referencia establecidos en ISO 8655; mientras que el segundo caso (sin hacer uso de la trampa de evaporación) en los tres resultados obtenidos parte del intervalo de medición se encuentra fuera de los límites de especificación, razón por la cual no es posible establecer la conformidad del resultado a un porcentaje de confianza del 95,45\% con un factor de cobertura $\mathrm{k}=2$, obligando en dos de los casos a reducir el intervalo de confianza para determinar un nuevo valor de confianza, y corriendo mayor riesgo en el uso del equipo.

Además, en los anexos se muestra el presupuesto de incertidumbre en cada caso estudiado, donde puede ser observado que cuando no se utiliza trampa de evaporación se tiene un aumento en el aporte por repetibilidad, reflejando lo importante del uso de este tipo de accesorios en la calibración de pipetas de pistón inclusive para valores de menos de $50 \mu \mathrm{L}$.

\section{CONSIDERACIONES FINALES}

Se demuestra de manera experimental y bajo las condiciones antes descritas que utilizar trampa de evaporación en la calibración de pipetas de pistón para volúmenes de hasta $100 \mu \mathrm{L}$ puede constituir un elemento de vital relevancia para determinar la conformidad de instrumentos volumétricos de dispensado. El no utilizar este dispositivo puede generar desviaciones de hasta un $1.3 \%$ inclusive, que se traduce en resultados altamente comprometedores en un análisis químico, medico y farmacéutico, entre otros.

Además se demuestra que en el dispensado de volúmenes mayores de $50 \mu \mathrm{L}$ (límite superior internacionalmente reconocido para el uso de trampa de evaporación), pueden existir desviaciones debido a la evaporación que comprometen los resultados y la evaluación de instrumentos como pipetas de pistón.

Considerando la incertidumbre de medición no se puede afirmar a un 94,45\% de confianza con $\mathrm{k}=2$ que los valores de volumen dispensado sin hacer uso de una trampa de evaporación están en conformidad con la referencia utilizada.

Debido a variaciones en la tasa de evaporación a razón de la saturación del aire circundante al área en la que se dispensa el fluido, temperatura del aire y presión atmosférica, es posible que se produzcan errores incluso superiores a los observados en la experimentación realizada. Inclusive la realización de corrección por efectos de evaporación puede llegar a ser insuficiente o poco precisa.

Se debe procurar, entonces, que el aire circundante al recipiente receptor del volumen se encuentre con humedad relativa cercano a la saturación completa; para este fin es apropiado el uso del dispositivo conocido como trampa de vapor, evitando así que el aire circundante tenga la capacidad de absorber agua del proceso de evaporación. En caso de no disponer de dicho dispositivo, se debe desarrollar una metodología que incluya el efecto de pérdida de volumen, asumiendo el posible error en la determinación del 
volumen dispensado como una fuente de incertidumbre y no como un valor de corrección, ya que generalmente los métodos de determinación de la tasa de evaporación son poco precisos y pueden tender al sesgo de los resultados obtenidos, siendo estas pérdidas por evaporación generalmente de una magnitud superior a los errores máximos permitidos por los procesos donde se requiere alta exactitud.

\section{AGRADECIMIENTOS}

Vicerrectoria de Investigación y Personal del Instituto de Investigaciones en Ingeniería-INII- y Laboratorio de Metrología Normalización y Calidad-LABCAL-.

\section{REFERENCIAS}

1. Batista, E., Pinto, L., Filipe, E., \& Van der Veen, A. M. H. Calibration of micropipettes: Test methods and uncertainty analysis. Measurement. 2007;40(3):338-42.

2. Majd, H. A., Hoseini, J., Tamaddon, H., \& Baghban, A. A. Comparison of the Precision of Measurements in Three Types of Micropipettes according to NCCLS EP5-A2 and ISO 8655-6. Journal of Paramedical Sciences. 2010;1(3).

3. Liang, D., Steinert, C., Bammesberger, S., Tanguy, L., Ernst, A., Zengerle, R., \& Koltay, P. Novel gravimetric measurement technique for quantitative volume calibration in the sub-microliter range. Measurement Science and Technology. 2012;24(2):025301.

4. ISO 8655-1/2/6, 2002, Piston-operated volumetric apparatus.

5. JCGM 104:2009. Evaluation of measurement data - An introduction to the "Guide to the expression of uncertainty In measurement" and related documents. (BIPM-IEC-IFCC-ILAC-ISO-IUPAC-IUPAP-OIML). 


\section{ANEXOS}

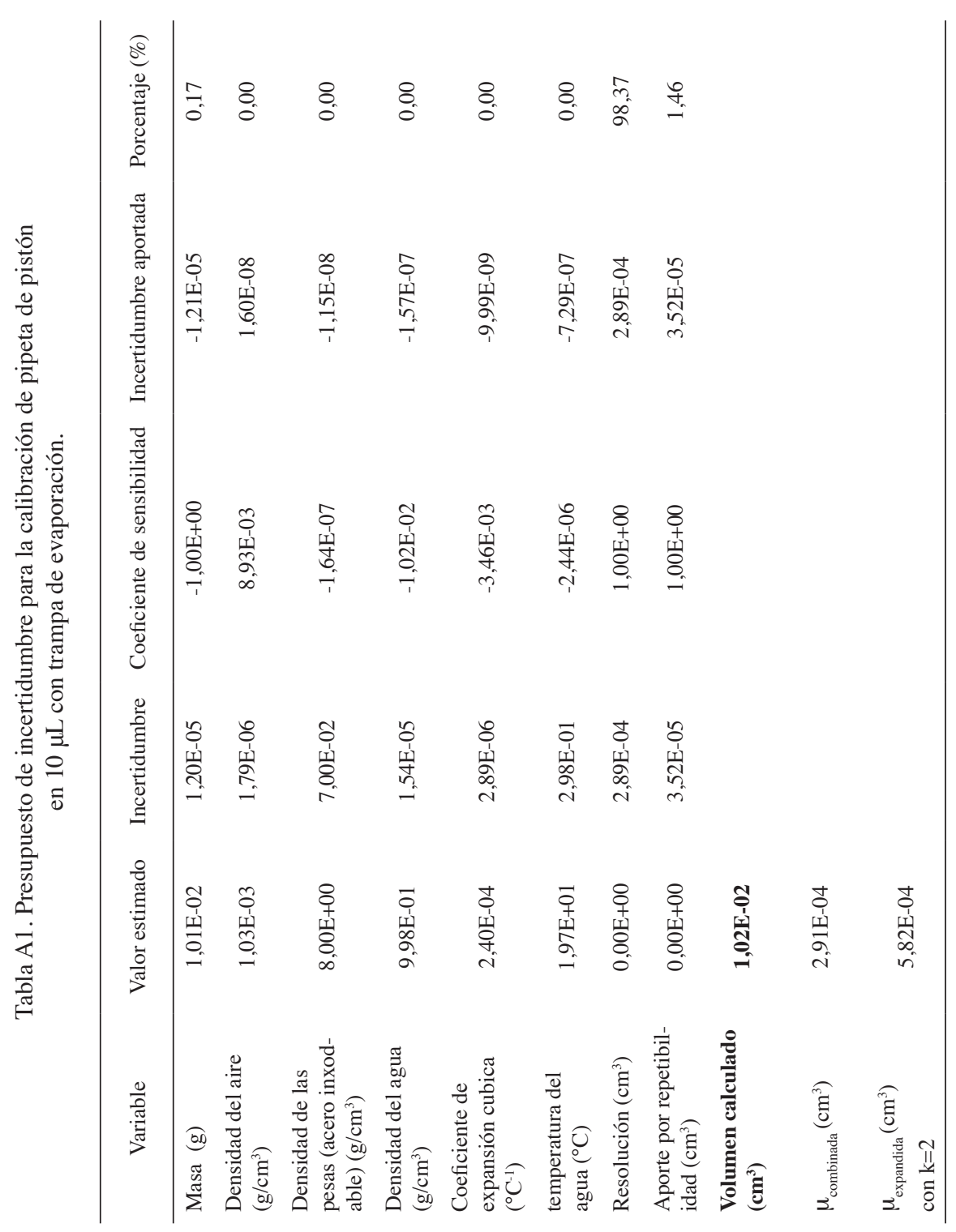




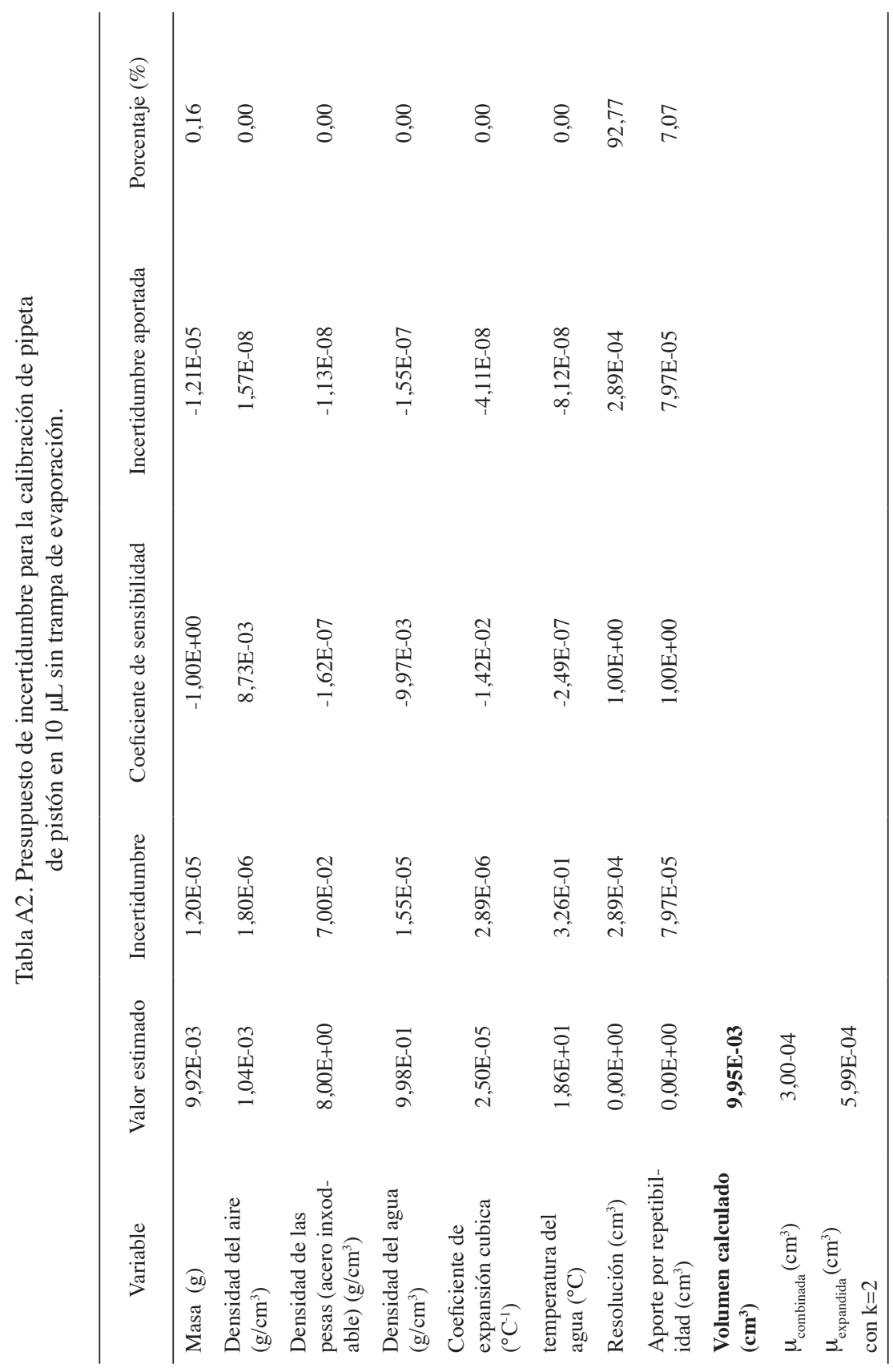


98 MÉNDEZ Y MARÍN: Evaluación del uso de trampa de evaporación en la calibración de pipetas de pipetas...

Tabla A3. Presupuesto de incertidumbre para la calibración de pipeta de pistón en $50 \mu \mathrm{L}$ con trampa de evaporación.

\begin{tabular}{|c|c|c|c|c|c|}
\hline Variable & $\begin{array}{c}\text { Valor } \\
\text { estimado }\end{array}$ & Incertidumbre & $\begin{array}{l}\text { Coeficiente de } \\
\text { sensibilidad }\end{array}$ & $\begin{array}{l}\text { Incertidumbre } \\
\text { aportada }\end{array}$ & Porcentaje (\%) \\
\hline Masa (g) & 4,94E-02 & $1,20 \mathrm{E}-05$ & $-1,00 \mathrm{E}+00$ & $-1,21 \mathrm{E}-05$ & 0,16 \\
\hline Densidad del aire $\left(\mathrm{g} / \mathrm{cm}^{3}\right)$ & $1,04 \mathrm{E}-03$ & $1,80 \mathrm{E}-06$ & $4,35 \mathrm{E}-02$ & $7,82 \mathrm{E}-08$ & 0,00 \\
\hline $\begin{array}{l}\text { Densidad de las pesas } \\
\text { (acero inxodable })\left(\mathrm{g} / \mathrm{cm}^{3}\right)\end{array}$ & $8,00 \mathrm{E}+00$ & $7,00 \mathrm{E}-02$ & $-8,05 \mathrm{E}-07$ & $-5,63 \mathrm{E}-08$ & 0,00 \\
\hline Densidad del agua $\left(\mathrm{g} / \mathrm{cm}^{3}\right)$ & $9,98 \mathrm{E}-01$ & $1,65 \mathrm{E}-05$ & $-4,97 \mathrm{E}-02$ & $-8,21 \mathrm{E}-07$ & 0,00 \\
\hline $\begin{array}{l}\text { Coeficiente de expansión } \\
\text { cubica }\left({ }^{\circ} \mathrm{C}^{-1}\right)\end{array}$ & $2,50 \mathrm{E}-05$ & $2,89 \mathrm{E}-06$ & $-1,34 \mathrm{E}-02$ & $-3,86 \mathrm{E}-08$ & 0,00 \\
\hline temperatura del agua $\left({ }^{\circ} \mathrm{C}\right)$ & $1,97 \mathrm{E}+01$ & $1,63 \mathrm{E}-01$ & $-1,24 \mathrm{E}-06$ & $-2,02 \mathrm{E}-07$ & 0,00 \\
\hline Resolución $\left(\mathrm{cm}^{3}\right)$ & $0,00 \mathrm{E}+00$ & $2,89 \mathrm{E}-04$ & $1,00 \mathrm{E}+00$ & $2,89 \mathrm{E}-04$ & 91,88 \\
\hline $\begin{array}{l}\text { Aporte por repetibilidad } \\
\left(\mathrm{cm}^{3}\right)\end{array}$ & $0,00 \mathrm{E}+00$ & $8,50 \mathrm{E}-05$ & $1,00 \mathrm{E}+00$ & $8,50 \mathrm{E}-05$ & 7,96 \\
\hline Volumen calculado $\left(\mathrm{cm}^{3}\right)$ & 4,96E-02 & & & & \\
\hline$\mu_{\text {combinada }}\left(\mathrm{cm}^{3}\right)$ & $3,01 \mathrm{E}-04$ & & & & \\
\hline $\begin{array}{l}\mu_{\text {expandida }}\left(\mathrm{cm}^{3}\right) \\
\text { con } \mathrm{k}=2\end{array}$ & $6,02 \mathrm{E}-04$ & & & & \\
\hline
\end{tabular}

Tabla A4. Presupuesto de incertidumbre para la calibración de pipeta de pistón en $50 \mu \mathrm{L}$ sin trampa de evaporación.

\begin{tabular}{|c|c|c|c|c|c|}
\hline Variable & $\begin{array}{l}\text { Valor } \\
\text { estimado }\end{array}$ & Incertidumbre & $\begin{array}{l}\text { Coeficiente de } \\
\text { sensibilidad }\end{array}$ & $\begin{array}{l}\text { Incertidumbre } \\
\text { aportada }\end{array}$ & Porcentaje (\%) \\
\hline Masa (g) & $4,88 \mathrm{E}-02$ & $1,70 \mathrm{E}-05$ & $-1,00 \mathrm{E}+00$ & $-1,71 \mathrm{E}-05$ & 0,12 \\
\hline Densidad del aire $\left(\mathrm{g} / \mathrm{cm}^{3}\right)$ & $1,03 \mathrm{E}-03$ & $1,80 \mathrm{E}-06$ & 4,29E-02 & $7,73 \mathrm{E}-08$ & 0,00 \\
\hline $\begin{array}{l}\text { Densidad de las pesas } \\
\text { (acero inxodable) }\left(\mathrm{g} / \mathrm{cm}^{3}\right)\end{array}$ & $8,00 \mathrm{E}+00$ & $7,00 \mathrm{E}-02$ & $-7,89 \mathrm{E}-07$ & $-5,53 \mathrm{E}-08$ & 0,00 \\
\hline Densidad del agua $\left(\mathrm{g} / \mathrm{cm}^{3}\right)$ & $9,98 \mathrm{E}-01$ & $2,83 \mathrm{E}-05$ & $-4,90 \mathrm{E}-02$ & $-1,39 \mathrm{E}-06$ & 0,00 \\
\hline $\begin{array}{l}\text { Coeficiente de expansión } \\
\text { cubica }\left({ }^{\circ} \mathrm{C}^{-1}\right)\end{array}$ & $2,40 \mathrm{E}-04$ & $2,89 \mathrm{E}-06$ & $-6,85 \mathrm{E}-03$ & $-1,98 \mathrm{E}-08$ & 0,00 \\
\hline temperatura del agua $\left({ }^{\circ} \mathrm{C}\right)$ & $1,99 \mathrm{E}+01$ & $4,68 \mathrm{E}-01$ & $-1,17 \mathrm{E}-05$ & $-5,49 \mathrm{E}-06$ & 0,01 \\
\hline Resolución $\left(\mathrm{cm}^{3}\right)$ & $0,00 \mathrm{E}+00$ & 2,89E-04 & $1,00 \mathrm{E}+00$ & $2,89 \mathrm{E}-04$ & 35,49 \\
\hline $\begin{array}{l}\text { Aporte por repetibilidad } \\
\left(\mathrm{cm}^{3}\right)\end{array}$ & $0,00 \mathrm{E}+00$ & $3,89 \mathrm{E}-04$ & $1,00 \mathrm{E}+00$ & $3,89 \mathrm{E}-04$ & 64,37 \\
\hline Volumen calculado $\left(\mathrm{cm}^{3}\right)$ & 4,89E-02 & & & & \\
\hline$\mu_{\text {combinada }}\left(\mathrm{cm}^{3}\right)$ & $4,85 \mathrm{E}-04$ & & & & \\
\hline $\begin{array}{l}\mu_{\text {expandida }}\left(\mathrm{cm}^{3}\right) \\
\text { con } \mathrm{k}=2\end{array}$ & $9,69 \mathrm{E}-04$ & & & & \\
\hline
\end{tabular}


Tabla A5. Presupuesto de incertidumbre para la calibración de pipeta de pistón en $100 \mu \mathrm{L}$ con trampa de evaporación.

\begin{tabular}{|c|c|c|c|c|c|}
\hline Variable & $\begin{array}{l}\text { Valor esti- } \\
\text { mado }\end{array}$ & Incertidumbre & $\begin{array}{l}\text { Coeficiente de } \\
\text { sensibilidad }\end{array}$ & $\begin{array}{l}\text { Incertidumbre } \\
\text { aportada }\end{array}$ & $\begin{array}{c}\text { Porcentaje } \\
\quad(\%)\end{array}$ \\
\hline Masa (g) & $9,93 \mathrm{E}-02$ & $1,20 \mathrm{E}-05$ & $-1,00 \mathrm{E}+00$ & $-1,21 \mathrm{E}-05$ & 0,13 \\
\hline Densidad del aire $\left(\mathrm{g} / \mathrm{cm}^{3}\right)$ & $1,03 \mathrm{E}-03$ & $1,80 \mathrm{E}-06$ & $8,74 \mathrm{E}-02$ & $1,57 \mathrm{E}-07$ & 0,00 \\
\hline $\begin{array}{l}\text { Densidad de las pesas (acero } \\
\text { inxodable) }\left(\mathrm{g} / \mathrm{cm}^{3}\right)\end{array}$ & $8,00 \mathrm{E}+00$ & $7,00 \mathrm{E}-02$ & $-1,61 \mathrm{E}-06$ & $-1,13 \mathrm{E}-07$ & 0,00 \\
\hline Densidad del agua $\left(\mathrm{g} / \mathrm{cm}^{3}\right)$ & $9,98 \mathrm{E}-01$ & $1,62 \mathrm{E}-05$ & $-9,98 \mathrm{E}-02$ & $-1,62 \mathrm{E}-06$ & 0,00 \\
\hline $\begin{array}{l}\text { Coeficiente de expansión } \\
\text { cubica }\left({ }^{\circ} \mathrm{C}^{-1}\right)\end{array}$ & $2,50 \mathrm{E}-05$ & 2,89E-06 & $-5,85 \mathrm{E}-02$ & $-1,69 \mathrm{E}-07$ & 0,00 \\
\hline temperatura del agua $\left({ }^{\circ} \mathrm{C}\right)$ & $1,94 \mathrm{E}+01$ & $3,83 \mathrm{E}-01$ & $-2,49 \mathrm{E}-06$ & $-9,53 \mathrm{E}-07$ & 0,00 \\
\hline Resolución $\left(\mathrm{cm}^{3}\right)$ & $0,00 \mathrm{E}+00$ & $2,89 \mathrm{E}-04$ & $1,00 \mathrm{E}+00$ & $2,89 \mathrm{E}-04$ & 73,77 \\
\hline Aporte por repetibilidad $\left(\mathrm{cm}^{3}\right)$ & $0,00 \mathrm{E}+00$ & $1,72 \mathrm{E}-04$ & $1,00 \mathrm{E}+00$ & $1,72 \mathrm{E}-04$ & 26,10 \\
\hline Volumen calculado $\left(\mathrm{cm}^{3}\right)$ & 9,95E-02 & & & & \\
\hline$\mu_{\text {combinada }}\left(\mathrm{cm}^{3}\right)$ & $3,36 \mathrm{E}-04$ & & & & \\
\hline $\begin{array}{l}\mu_{\text {expandida }}\left(\mathrm{cm}^{3}\right) \\
\text { con } \mathrm{k}=2\end{array}$ & $6,72 \mathrm{E}-04$ & & & & \\
\hline
\end{tabular}

Tabla A6. Presupuesto de incertidumbre para la calibración de pipeta de pistón en $10 \mu \mathrm{L}$ sin trampa de evaporación.

\begin{tabular}{|c|c|c|c|c|c|}
\hline Variable & $\begin{array}{c}\text { Valor } \\
\text { estimado }\end{array}$ & Incertidumbre & $\begin{array}{l}\text { Coeficiente de } \\
\text { sensibilidad }\end{array}$ & $\begin{array}{l}\text { Incertidumbre } \\
\text { aportada }\end{array}$ & Porcentaje (\%) \\
\hline Masa (g) & $9,82 \mathrm{E}-02$ & $1,70 \mathrm{E}-05$ & $-1,00 \mathrm{E}+00$ & $-1,71 \mathrm{E}-05$ & 0,17 \\
\hline Densidad del aire $\left(\mathrm{g} / \mathrm{cm}^{3}\right)$ & $1,04 \mathrm{E}-03$ & $1,84 \mathrm{E}-06$ & $8,64 \mathrm{E}-02$ & $1,59 \mathrm{E}-07$ & 0,00 \\
\hline $\begin{array}{l}\text { Densidad de las pesas } \\
\text { (acero inxodable) }\left(\mathrm{g} / \mathrm{cm}^{3}\right)\end{array}$ & $8,00 \mathrm{E}+00$ & $7,00 \mathrm{E}-02$ & $-1,60 \mathrm{E}-06$ & $-1,12 \mathrm{E}-07$ & 0,00 \\
\hline $\begin{array}{l}\text { Densidad del agua }(\mathrm{g} / \\
\left.\mathrm{cm}^{3}\right)\end{array}$ & 9,98E-01 & $1,55 \mathrm{E}-05$ & $-9,88 \mathrm{E}-02$ & $-1,53 \mathrm{E}-06$ & 0,00 \\
\hline $\begin{array}{l}\text { Coeficiente de expansión } \\
\text { cubica }\left({ }^{\circ} \mathrm{C}^{-1}\right)\end{array}$ & $2,50 \mathrm{E}-05$ & 2,89E-06 & $-9,45 \mathrm{E}-02$ & $-2,73 \mathrm{E}-07$ & 0,00 \\
\hline temperatura del agua $\left({ }^{\circ} \mathrm{C}\right)$ & $1,90 \mathrm{E}+01$ & $3,55 \mathrm{E}-01$ & $-2,46 \mathrm{E}-06$ & $-8,73 \mathrm{E}-07$ & 0,00 \\
\hline Resolución $\left(\mathrm{cm}^{3}\right)$ & $0,00 \mathrm{E}+00$ & 2,89E-04 & $1,00 \mathrm{E}+00$ & 2,89E-04 & 48,46 \\
\hline $\begin{array}{l}\text { Aporte por repetibilidad } \\
\left(\mathrm{cm}^{3}\right)\end{array}$ & $0,00 \mathrm{E}+00$ & 2,97E-04 & $1,00 \mathrm{E}+00$ & 2,97E-04 & 51,37 \\
\hline $\begin{array}{l}\text { Volumen calculado } \\
\left(\mathrm{cm}^{3}\right)\end{array}$ & 9,85E-02 & & & & \\
\hline$\mu_{\text {combinada }}\left(\mathrm{cm}^{3}\right)$ & $4,15 \mathrm{E}-04$ & & & & \\
\hline $\begin{array}{l}\mu_{\text {expandida }}\left(\mathrm{cm}^{3}\right) \\
\text { con } \mathrm{k}=2\end{array}$ & $8,29 \mathrm{E}-04$ & & & & \\
\hline
\end{tabular}

